\title{
Understanding Sexual Reproductive Health from the perspective of Older Adolescents in Northern Thailand: A Phenomenological study
}

\section{Panitsara Leekuan}

University of Phayao

Ros Kane ( $\nabla$ rkane@lincoln.ac.uk)

University of Lincoln - Brayford Campus: University of Lincoln https://orcid.org/0000-0002-6926-2530

Panpimol Sukwong

University of Phayao

Waratya Kulnitichai

University of Phayao

\section{Research Article}

Keywords: Older adolescence, Sexual Health, Reproductive Health, Sexual and Reproductive Health Education, Phenomenology

Posted Date: February 10th, 2022

DOI: https://doi.org/10.21203/rs.3.rs-1323664/v1

License: (c) (i) This work is licensed under a Creative Commons Attribution 4.0 International License. Read Full License 


\section{Abstract}

Background: Worldwide, Sexual Reproductive Health $(\mathrm{SRH})$ issues comprise a third of health problems for women aged 15-44. SRH education equips people with knowledge of concepts around sexuality and reproduction, and the attitudes and skills help to make informed decisions to prevent sexual and reproductive ill-health problems, including unplanned pregnancy and HIV/AIDS, and other sexually transmitted infections (STIs). The aim of this study is to explore the experiences of older adolescents relating to $\mathrm{SRH}$, examining their attitudes toward sex and contraception, and to identify the gaps in decision-making to prevent risk-taking behaviour.

Methods: A qualitative phenomenological study was undertaken with 30 adolescents aged 18-19, purposively and snowball sampled from a university in Northern Thailand from July 2020 to January 2021. In-depth individual interviews were conducted until data saturation was reached. Data were recorded, transcribed, and analysed in ATLAS.ti version 9, using Modified Interpretative Phenomenological Analysis to identify related themes.

Results: Participants revealed five key experiences of SRH related to sex and contraception: Keeping a secret, Seeking Freedom and Love; Having SRH education; Self-protection; Parental acceptance. All findings reflected the value and impact of SRH on the experiences of older adolescents.

Conclusions: This study provides detailed knowledge about adolescents' perspectives of SRH and rights in terms of accessing sexual and reproductive health care and information as well as autonomy in sexual and reproductive decision-making. Gaining SRH education can assist decision-making concerning contraceptive methods for family planning and STI prevention. The study recommends that SRH and rights should be designed responsively and appropriately for female and male adolescents, their families, and society. The inclusion of SRH issues should be informed and advocated healthcare providers, educators, policy makers, and systems to empower adolescents in order to achieve effective SRH education.

\section{Plain English Summary}

Worldwide, Sexual Reproductive Health issues make up a third of health problems for women aged 15-44. Sexual Reproductive Health education helps people to gain knowledge around around sexuality and reproduction, and the attitudes and skills help to make informed decisions to prevent sexual and reproductive health problems, including unplanned pregnancy and other sexually transmitted infections. This research aimed to explore the experiences of older adolescents relating to Sexual Reproductive Health, examining their attitudes toward sex and contraception, and to identify the gaps in decisionmaking to prevent risky behaviours.

We interviewed 30 adolescents aged 18-19, from a university in Northern Thailand from July 2020 to January 2021. The interviews were recorded and the recordings typed and analysed to identify the following related themes: Keeping a secret, Seeking Freedom and Love, Having SRH education; Self- 
protection; Parental acceptance. All findings reflected the value and impact of Sexual Reproductive Health on the experiences of older adolescents.

This study provides detailed knowledge about adolescents' perspectives of Sexual Reproductive Health and rights in terms of accessing sexual and reproductive health care and information as well as autonomy in sexual and reproductive decision-making. Gaining Sexual Reproductive Health education can assist decision-making concerning contraceptive methods for family planning and the prevention of sexually transmitted infections. We recommend Sexual Reproductive Health issues should be informed and advocated by healthcare providers, educators, policy makers, and systems to empower adolescents in order to achieve effective Sexual Reproductive Health education.

\section{Background}

Adolescents are in a period of transition from childhood to adulthood, facing profound physiological and psychological changes and challenges (1). The period of adolescence can be frustrating; both cognitive and social development can be painful, traumatic, excruciating, and unsatisfactory (2). The onset of adolescent development brings physical and physiological changes, as well as new vulnerabilities, sometimes including human rights abuses, particularly in the areas of sexuality, marriage, and childbearing (3). As a result, adolescents face a range of health and social challenges as they mediate the biological and social transition into adulthood.

The experiences of older adolescents (aged 18-19 years) vary greatly by gender, race, ethnicity, socioeconomic status, and other factors that shape the way in which they respond to their physical and emotional development and assumption of the roles of adulthood. People commonly develop a deeper sense of identity during late adolescence, such as a personal sense of gender and sexuality, establish values about sexual behaviour and develop skills for romantic relationships (4). Sexual relationships for adolescents mean early entrance to the world of adulthood and potential parenthood. Lacking adequate knowledge and skills about developing sexual relationships can make adolescents vulnerable to a higher risk of unintended pregnancy, unsafe abortion and sexually transmitted infections (STIs), including HIV/AIDS (5).

The ultimate objective of sexual health not only concludes the attainment of physical, emotional, mental and social well-being in relation to sexuality, but it also focuses on the absence of disease, dysfunction or infirmity (6). A positive and respectful approach to sexuality and sexual relationship as well as the possibility of having pleasurable and safe sexual experiences, free of coercion, discrimination and violence are keys to sexual health and well- being (7). Additionally, sexual rights which embrace certain human rights at the international and region level as well as national laws must be respected, protected and fulfilled for all people in order for the highest attainable standard of health $(8,9)$. These are a necessary condition attaining sexual health. Rights critical to the realization of sexual health include: the right to health, the right to be free from discrimination, the right to privacy, the right not to be subjected to

torture or ill-treatment, the right to determine the number and spacing of one's children, and the right to be 
free from sexual violence $(9,10)$. More recently, the World Health Organization (WHO) works to promote and protect everybody, including adolescents to achieve their full potential for sex and reproductive health and well-being in order to meet the needs of diverse populations, particularly the most vulnerable (11).

Adolescent sexual and reproductive health (ASRH) in low and middle income countries is a major public health challenge $(5,11)$. WHO $(5)$ report that over 1 million adolescents contract a Sexual Transmitted Infection every day worldwide. Globally, almost half of new HIV infections occur in men and women aged 15-24. All sexual infections cause a huge health and economic burden, especially in low- and middleincome countries where they account for $17 \%$ of economic losses caused by ill-health. Adolescents are a high-risk group who face barriers to access accurate information about their health and rights and how to protect themselves from pregnancy and sexually transmitted infections (STIs) (12). Comprehensive sexuality education plays a crucial role in empowering young people to know and exercise their rights, including the right to delay marriage and the right to refuse unwanted sexual advances.

There are an estimated 580 million adolescent girls in the world today, and $88 \%$ of them live in lowincome and middle-income countries (LMICs) (8). Sexual and reproductive health (SRH) continues to elude many, and many are denied the right to make safe and informed decisions that affect their health and wellbeing (13). Clearly, SRH is only one among the many dimensions of adolescent girls' health, including notably nutrition and mental health, and improvements in SRH depend on progress in other dimensions of health; at the same time, girls' SRH situation has huge implications both for their later health as well as the health of the next generation (13). As the UN reviews 20 years of implementation of the ICPD Programme of Action (14) and designs a new global agenda for 2015 and beyond, it is important to review the situation of today's cohort of adolescent girls and assess their need for sustained and expanded national and global attention and investment.

There are various public health concerns worldwide caused by high-risk behaviour among adolescents, such as early sexual initiation, multiple partners, and unprotected sexual intercourse. The United Nations Population Fund reported there has been a 25 per cent increase in the global contraceptive prevalence rate in worldwide (15). As a result of this, it is internationally recognised that the adolescent birth rate has decreased steeply and the maternal mortality ratio has declined. However, the improvement has been slow and varied by country. This is because an estimated 100 million women worldwide are still not using safe and effective family planning methods to prevent unwanted and unintended pregnancy and a new global target (SDG 3.1) on reducing maternal death have been not met (11) One of five critical targets to help countries in reducing preventable maternal deaths is that $65 \%$ of women to be able to make informed and empowered decisions regarding sexual relations, contraception use and their reproductive health. Therefore, urgent action is needed to improve the health and survival of women and babies and assist countries greater equality and coverage at the national and subnation level (11).

Thailand, as an upper middle-income economy, is faced with an increasing number of adolescent pregnancies and the rate of new Sexual Transmitted Infections, including HIV/ AIDS (16). These increasing of statistics have been attributed to the consistently falling prevalence of contraceptive use 
(16). The national survey on the prevalence of consist condom use at the last sex among young people and adult aged $15-24$ years found that merely $60 \%$ had consistently used condoms (17). According to a study (18) in Thailand uncovered the highest prevalence and determinants of contraceptive use among unmarried adolescents which was $75.8 \%$ of secondary school adolescents used contraception during sexual intercourse. Of these, $84 \%$ consistently used condoms, followed by emergency pills and the calendar method. Using contraception among unmarried school-going adolescents is very crucial to maintain a stable balance between their sexual and reproductive health. Therefore, adolescents could be promoted to use contraception and mentally prepared to practice safe sex. Typically, young people are fully developed in their sexual identity by this stage (19). They may also live independently from their families and may take on adult responsibilities and roles (20). Late adolescents are able to understand the consequences of current actions and are very concerned about their future, career goals, and often consider desirable potential spouses or life-partner related to the transition to the adult role $(19,21)$. Consequently, they speedily develop the ability to make independent decisions and to compromise. These trends can also encourage adolescents to take pride in their work and be self-confident.

Adolescents considered to be in older adolescence (ages 18-19 years) are targeted because of the likelihood that they have increased cognitive ability and psychological development that allow clear decision-making regarding participation and discussion of sensitive topics (Peterson et al., 1995). They also face novel challenges, including engagement in romantic and sexual relationships (22). Previous studies have discovered the experiences of pregnant adolescents and revealed that contraceptive decision-making is influenced by sexual health education, especially knowledge about contraception and adolescents' perception regarding hormonal contraception as well as gender power imbalance $(23,24)$. Gender role is one of various factors involving adolescent pregnancy $(1,15,24,25)$.

Young women inclined to engage in sexual relationship for showing love or long-term relationship, but young men engaged in sexual relationship for curiosity or the need to satisfy sexual drive (26). Moreover, the adverse consequences of sexual activity among young women were unwanted pregnancy, sexual violence, or partner abandonment. These young women also experienced unequal gender relations influenced on their sexual health $(1,24,27)$.

To be effective, adolescent male should be involved in sexual reproductive health experience and addressed men's behaviours in their various roles as well as their reproductive health and rights as human beings. Young men also lacked really understand and sexual health education as well as inform prevention messages and services. In order to prevent pregnancy and the risks of STIs and HIV/ AIDS, accessing and using contraception enables adolescents and their partners to exercise their rights to decide freely and responsibly the number and spacing their children and to have the information, education and means to do so. Enhancing sexual reproductive health education is therefore a vital issue among older adolescents, as this can assist in preventing adverse outcomes of sexual relationship such as sexual transmitted infections or unintended pregnancy (5). Understanding sexual reproductive health experience and its impacts on practices among older adolescents both genders are crucial for promoting 
sexual reproductive health and rights in the transition to adulthood as well as opportunities for improving sexual and reproductive health care services and interventions.

Due to the private and sensitive nature of the subject, relatively little is known about SRH among those in older adolescence and their knowledge, attitudes, and practices, particularly in Northern Thailand. To address this gap and provide data for improving understanding SRH experience and inform prevention message and services to female and male university students, this study conducted an interpretive phenomenological study to really understand and describe late adolescent experience of SRH in order to generate evidence relevant to a wide range of SRH programmes for adolescents and practitioners.

\section{Methods}

- The aim

The aim of this study is to explore the experiences of older adolescents relating to $\mathrm{SRH}$, examining their attitudes toward sex and contraception, and to identify the gaps in decision-making to prevent risk-taking behaviour.

- Study design

Interpretative phenomenology, as developed by Heidegger $(28,29)$, was selected as the research methodology, in order to uncover the meanings older adolescents give to their experiences of SRH.

- Study Setting

The study was conducted in a university in the Northern Thailand province. In this study, the sampling must enable proper access to the experiences of adolescents who are involved with sexual reproductive health in terms of sex relationship, in line with phenomenological study. Potential participants were subject to the inclusion criteria of being adolescents aged 18-19 years referred as older adolescents. In comparison to early and middle adolescence, adolescents in this group are more composed and mature. Not only adolescents have already acquired major physical changes, but they have also obtained cognitive maturity. Adolescence features like risk taking, curious, anxious are less prevalent among late adolescence. As a result of this, late adolescence is also perceived as the period of opportunity $(1,30)$.

University students are currently pursuing higher education and have achieved the task of late adolescent development to early adulthood or middle adulthood (31). Based on the agent, the main developmental task of university students is to mature themselves (32). University students also tend to have emotional stability and more critical thinking. In Arnett's emerging adulthood theory (33), University students with an age range of 18-19 years are still in the process of finding their identity as evidenced by being still in the study period, still looking for a permanent job, and not fully independent from their parents. In this context, University students have higher demands and increasingly difficult assignments as well as stress triggers. 
To recruit participants, this study employed two different kinds of sampling: purposive and snowball sampling. For the phenomenological approach, purposive sampling is suitable because the goal of this study was to discover the experience of adolescents who were involved with reproductive health.

Snowball sampling involves collecting data on few adolescents of target population who can be reached initially, then asking those participants to help recruit others from their social network. This is appropriate when adolescents of the target population are hard to reach or difficult to located, as these participants have proven to be. Hence, sampling was terminated at the point of redundancy when no new information was forthcoming from participants.

- Data collection

The researcher acquainted with two volunteers who worked as student services volunteer who were familiar all adolescents. They assisted in initial recruitment and contacted the eligible informants and gave them research information documents via phone and email as well as referred potential participants to the first researcher (LP). Eligible criteria were: (a) 18-19 years of age, (b) had experience of sexual relationship, (c) Thai speaking (d) willing to be interviewed and digitally recorded, and (f) provided informed consent. Exclusion criteria were: (a) left from the university during the study and (b) cognitive impairment.

The initial 5 individuals, who were referred from two volunteers, expressed interest in participants via phone. The first researcher checked them based on their fit with a purposive sampling strategy, which involved selecting participants deliberately based on the specific qualities they possess. The first researcher (LP) made appointment with the participants via phone prior to the interview. Interviews were conducted in adolescents' accommodation and ranged in length from 60-90 minutes. Data collection involved a face -to- face unstructured interview in the Thai language with the participants between July 2020 and January 2021 via a secure web-based platform (Microsoft Teams) due to the Covid-19 pandemic, which was physical distancing and social isolation. At the end of each interview, the first researcher thanked the participant and asked her/ him to help recruit further participants who had the same experience from their social network. Thus, the next participants can continue to interview as data collection and those had all been recruited through snowballing and had therefore expressed a particular in this study. Data were collected until analytical saturation was reached and no new insights emerged after 30 participants were interviewed.

\section{- Data Analysis}

Initial data analysis took place alongside data collection and interviews continued until 30 saturations has been reached and variety to answer the research question. Interview data were transcribed verbatim in Thai. The transcripts were read and re-read alongside the first author's field notes to gain sense of each adolescent's experience as a whole. The analysis continued process of discussions between three authors (LP; PS; WK) in order to follow the Heideggerian principle. To describe the experiences of SRH among late adolescents, the first researcher analysed the data following Modified Interpretative 
Phenomenological Analysis outlined by Heidegger (28), Packer (34, 35), van Manen (36) and Smith et al. (37):

Following Packer (34) (p. 1090), Initial read and reread each interview transcript and listened to the audiorecordings in order to achieve data immersion and identify meaning units (36). Next, the interpretive processes were highlighted and assigned codes or phases. The coding meaning units were merged and developed into sub-themes concerning the same concepts in relation to particular phenomena. Similar sub-themes were identified links and developed as well as refined to themes in order to interpret participants' experiences. Analysis was done in Thai and translated to English at the point of publication.

Table 1

Table 1

Coding process - meaning units, sub-themes and main themes

\begin{tabular}{|c|c|c|}
\hline Meaning units & Sub-themes & Themes \\
\hline $\begin{array}{l}\text { I remembered that condoms could prevent sexual diseases. I disliked } \\
\text { taking pills and feared implants, and contraceptive injections. } \\
\text { However, if I didn't use any contraceptive methods, I would face } \\
\text { unplanned pregnancy and be out of schooling. I don't want to stop } \\
\text { schooling due to pregnancy. We might face with some problems, such } \\
\text { as raising children and finance. [RH 18] }\end{array}$ & $\begin{array}{l}\text { Focusing on } \\
\text { the risk of } \\
\text { diseases and } \\
\text { consequences }\end{array}$ & $\begin{array}{l}\text { Having } \\
\text { SRH } \\
\text { education }\end{array}$ \\
\hline $\begin{array}{l}\text { When I had sexual intercourse and used condoms, there was an } \\
\text { accident of a broken condom. I felt fear and stress. I did not know how } \\
\text { I could solve this problem. I then took emergency pills at first. I also } \\
\text { waited for my period. After that, it came. I therefore felt relieved. [RH } \\
\text { 25] }\end{array}$ & $\begin{array}{l}\text { Using } \\
\text { contraceptive } \\
\text { methods }\end{array}$ & \multirow[t]{2}{*}{$\begin{array}{l}\text { Self- } \\
\text { protection }\end{array}$} \\
\hline $\begin{array}{l}\text { My partner asked for having sexual intercourse with me. I then asked } \\
\text { him about condoms. He drank alcohol and did not prepare a condom. } \\
\text { I said "no". He was angry. I reasserted my choice and tried to explain } \\
\text { more about the negatives of non-use of any contraceptive methods. If } \\
\text { we made the mistake, we might have some problems. [RH 26] }\end{array}$ & Negotiation & \\
\hline
\end{tabular}

\section{Results}

The participants in this study comprised 30 adolescents ( 3 men and 27 women). 17 participants were aged 18 years and 13 participants were aged 19 years. In terms of marital status, 14 participants were cohabiting with their partners and 16 participants were single.

The five identified themes reflected the phenomenological interpretation of adolescents' experiences of SRH: Keeping a secret, Seeking Freedom and Love, Having SRH education, Self-protection, and Parental acceptance.

\section{Keeping a secret}


Engaging in a sexual relationship with someone of the opposite sex among adolescents causes disapproval from some parents and families. Many participants contrived not to tell their parents or families of their relationships with their partners. A simple reason for secrecy was the feeling of unease in exposing their relationships for fear of this resulting in parents' disappointment.

My dad didn't know about my relationship with my partner. This was because he didn't want me to have a boyfriend. He also wanted me to graduate studying before having a boyfriend because he worried about a social stigma. My dad had more authority to decide everything in my family. I fear him when I had to ask him for permission. I scared him the most. I then decided to keep my relationship as a secret. He also asked me that "What would you select between studying or having a boyfriend" I answered that I chose studying. [RH 21]

\section{Seeking for Freedom and Love}

Most of participants in this study searched for freedom from parental strict rules and regulations. They felt excited when they left their houses for their educational setting. This seems to be a new world for them to seek for new friends and new environment around them. For some participants, they described their personal encounter in seeking love and an act of love on their part at that particular time. When they felt close to partners, they reported feeling happier and even healthier. The participants felt loved, understood, and safe in her new relationship.

Our first relationship took place when he bought some food and brought it to me. He also took care of me as well. Nowadays, we live in the flats outside the university, but we are living separately. Our flat are opposite. However, he often comes to see me and pick me up for studying. This makes me feel that I receive the love from him all time that I try to find it. I think that it is giving love together. I also appreciate with our relationship and his activities. [RH 03]

\section{Intimacy relationship}

An intimate relationship, including physical, emotional, and sexual intimacy, can lead to having full sexual activity. Among the reasons for having a sexual relationship, adolescents seek love, acceptance, and recognition. The majority of female participants expressed a desire to be worthy of recognition and love, and they believed that living together and having sexual relationships could maintain their relationships with their partners.

Our relationship seems like a close-friend relationship. He is a friend who can discuss every story with me. He also took care of me and his concern has always been about my activities. This made me feel that there was someone to care for me and love, apart from my parents. I felt better with my life. I also did not worry about his behaviour. I was then happy and studies efficiently. [RH 01]

\section{Feeling endearment}


Most participants reported that intimacy promoted feelings of endearment, when their partners took care of them. This also reflected feelings of love and trust, honesty, and comfort that came with having intimacy.

I felt that he loved me when he took care of me and sent me to study every morning. We love together. I think that is giving love to each other. We had only time to live together, chat and confide in someone. These were my happiness. [RH 03]

\section{Openly sharing feelings}

Building and maintaining intimacy in a relationship depends on open communication about feelings and desires in the relationship. This also includes sharing thoughts and feelings openly to raise the level of emotional intimacy. Participants exposed how they felt during cohabitation and having sexual relationships.

I am an extrovert. It was not wrong if we could talk openly together. Finally, we accepted each other. In the initial time, we did not dare to talk face-to-face. I sometimes felt pain when we had sex activity. I therefore told him. I think that non-verbal communication is not good, because others do not know how you think and what you want. We should learn about each other. [RH 03]

\section{Having SRH education}

$\mathrm{SRH}$ education is axiomatically lays an important role in sexuality education programmes; in particular, it renders helpful knowledge and shapes attitudes towards human development, sexuality, relationships, gender roles, and decision-making about sexuality (38). It also breaks barriers in the prevention of adolescent pregnancy (39). The integration of sexuality education in the curriculum provides a broad and rich channel of information about a variety of sexuality-related issues, from the growth and development of the human body and reproductive physiology to the development of healthy sexual attitudes and values.

The majority of participants perceived that SRH education was an important for learning and good health, as well as for decision-making to use contraceptive methods. SRH education also assists to control behaviours and reiterate the deficiency of correct and easily accessible SRH information sources. Participants also indicated that they learned about contraception from school, the internet, friends, family, and health providers, but schools, universities, and health providers were the most frequently cited.

During studying in the secondary school and the high school, I attended a training programme specially designed for adolescents about SRH education. It plays a crucial role for youth and is compulsory in the schools. I also learned about the risk of sexual transmitted diseases and HIV/AIDs and contraception in General Education in the University. Additionally, I saw it in the movies and in YouTube and in any video clip about the risk of sexual transmitted diseases and HIV/AIDs, and prevention from unintended pregnancy. As a result, this can increase my knowledge about the results of sexual relationship problems 
and brush up, as well as repeat warnings about preventing these diseases. I believe that studying provides new knowledge, though it is repeated again and again. [RH 06]

$\mathrm{SRH}$ was mainly discussed in terms of the risk of diseases and consequences of not using contraceptive methods, particularly STIs. SRH education influenced contraceptive decisions, due to the desire to prevent STIs and consequences such as dropping out of schooling due to unplanned pregnancy, and financial problems. One participant stated that she obtained SRH education related to the risk and the consequences of unprotected sexual intercourse from many sources.

In the secondary school, some health providers from a hospital provided me with education about contraceptive methods, such as the contraceptive implant, condoms, and oral pills, as well as protection of the risk of sexual relationship. I also studied in General Education about the risk of sexual transmitted diseases and HIV/AIDs and contraceptive methods, as well as the risk of having sexual relationship in several special occasions such as Valentine's Day or Loy Kra Thong's Day. [RH 01]

\section{Self-protection}

Having SRH education in adolescence can increase awareness about prevention from the risk of STIs by using contraceptive methods. The awareness about different contraceptive methods, including condoms, hormonal methods (i.e., oral pills, implants, and contraceptive injections), and withdrawal can assist adolescents to suitably utilise choices for them and their partners.

\section{Using contraceptive methods}

Both male and female participants revealed that the most commonly used contraceptive methods were male condoms and oral contraceptive pills. Some of them had experienced accidents (e.g., a broken male condom), as a result of which they used combined approaches (i.e., emergency oral pills).

I asked my partner to use condom when we had sexual relationship. I sometimes bought oral pills if he did not prepare male condoms. I think that protection of the risk of sexual transmitted diseases and HIV/AIDS is a crucial issue for us, me and my partner. We have love and must protect ourselves [RH 02]

\section{Negotiation}

Female participants disclosed they negotiated to use condoms prior to having sexual activity, in order to achieve safe sex. Their priority was preventing the risk of STIs and unintended pregnancy.

I always ask him before having sex about condom. When he didn't have it, I said "No". Although he tried to persuade me, I confirmed the same word. He then felt frustrated and went back to his house. I refused him when he requested to have sexual relations without using a condom. He accepted my decision. [RH 03]

\section{Concern for the family}


To address concern for their families, participants attempted to avoid unintended pregnancy and the risk of Sexual Transmitted Infections. The vast majority of participants attended sexuality education and so they were keenly aware of the potential lifelong consequences of unprotected sexual activities.

It is not only that I love him, but also there are many people in our families who love us. If I was ill or pregnant due to having sex without protection, they would regret. I am also a university student; therefore, I am not ready to be a housewife or a mother. I think of my parents. They raised two daughters, and they might not want to have a grandchild at this time. We might have financial problems. [RH 02]

\section{Parental acceptance}

Premarital sex is a common practice among young people in many countries, including in Thai society. This pattern varies substantially across generations and gender. Rapid change in Thai society and culture is reflected in changing sexual values and mores. The family, school, and society view unplanned pregnancy, abortion, and sexualized media with disapprobation. The family is a significant factor in the development of sex behaviour among adolescents. Participants described their own parents and partner's parents approaches about their relationships. One participant opined that both families had an opportunity of getting to know each other, as a result of which they trust their children.

My parents expected about my future because I was the oldest daughter. My parents therefore wanted me to grow up as a good adult in the society and to prevent from unplanned pregnancy. Initially, my mother did not approve about our relationship. We met together via Facebook around a year before becoming lovers. After that, he and his parents came to see my family. Both families accepted our relationship. [RH 01]

\section{Openness}

Mothers were reported to be more frequent communicators about sexuality than fathers, and daughters received more parental communication than sons. Adolescent perceptions of the openness of family communication are the significance of subjective meaning in communication and relationships. One adolescent who perceived her communications as open and problem-free with her mother considered that this influenced her decision-making about taking care for herself and preventing sexual health problems.

I felt better when I told my parents everything openly, and they listened to my voice, such as private stories or studying. This has taken place from the past until now. Therefore, when we faced some problems, our parents gave some suggestions. My parents were initially concerned about my relationships with my partner. My mother told me that you should rethink about the relationship, but she did not blame me. She always tells me about caring for myself and protecting from the risks of sexual activities, such as unintended pregnancy and diseases. [RH 17]

\section{Trustfulness}


Trustfulness refers to the belief of the honesty and reliability of the others, including their decisions and actions. Trustfulness also provides a positive basis for such relationships. One of the exemplars described her mother who believed in her behaviours and decisions about her relationship with her partner.

I and my mother always talk about every story together. I told her that I had a boyfriend. She gave me many suggestions. When we have some problems, she then tried to come down. She knew about our relationship and having a sexual relationship. However, she did not blame me, but she suggested me to use contraception. She also motivated me to look ahead. [RH 20]

\section{Discussion}

The research findings provide insights into the SRH experiences of late adolescent university students in the context of Northern Thailand. The adolescents' accounts underlying SRH experiences about sexual relationships indicated motivations for having SRH education. The findings uncovered how these adolescents comprehend their SRH education and made decisions to use contraceptive methods in order to avoid the risk of STIs and unintended pregnancy. There is evidence of competence and confidence which is a great achievement. The key of this study is not to sustain this and also be mindful for future generations as well as may be other populations of older adolescents.

\section{Adolescent Love and Romantic relationships}

Making sense of their experiences, the participants in this study exposed they sought for love. They had also looked elsewhere for satisfying relationship to be their own world. Thinking that they had found love with their partners involved sexual intimacy. With seeking love in adolescence, the adolescent development in seeking love and romantic relationships identified in the current study were broadly consistent with developmental theories $(40,41)$. Previous studies on love and romantic relationship in adolescence found that middle to late adolescence worried about maintaining and sustaining relationships when intimacy levels, emotional investment, skills and commitment in romantic experiences increase across all developmental stages of adolescence (41).

Adolescent romantic relationships can not only improve growth, resilience, and happiness, but can also engage in sexual development which is an important part of growing to adulthood. Most of participants believed that having boyfriend or girlfriend can enhance one's confidence. They were also happier with themselves with support, trust, and closeness in romantic relationships. Similarly, qualities of adolescent romantic relationship can predict relative changes in mental health developed to adulthood (42). This mirrors findings of the link between supportive partner and increased mental health development.

Romantic relationships are an important aspect of individual development and socialisation, especially in adolescent development. Through romantic relationships, individuals learn intimacy and crucial interpersonal skills. Romantic relationships can play a positive role in adolescent development (43), presenting an emerging development task (in terms of psychological development), which is an 
important domain in which to explore and gain experience (44). Romantic relationships are related to the level of closeness, depth of feeling, consideration of characteristics, the intensity of communication, type of activity, and physical contact between partners, with long-term implications with regard to life partners and future relationships and associated holistic well-being (45).

Adolescents commonly experience their first romantic relationships and may experience overpowering emotions associated with falling in love, which can lead to having sexual relationships despite rational objections to this. Relationships in which sexual activity is the primary aspect can also arise, such as transactional sexual encounters, which have various socio-economic, sexual, and psychological reasons and impacts. This study illustrated the feelings of romantic love associated with enhancing happiness and life satisfaction in adolescent relationships with increasing maturity. Wheeler et al. (46) reinforced that the nature of romantic relationships during late adolescence and young adulthood can shed light on many aspects of adolescent development, including the distinctive developmental contexts of intimate relationships.

Similarly, Kansky and Allen (42) revealed a link between romantic relationships with supportive partners and future intimacy relationships and well-being. Adolescent romantic relationships have the potential to affect psychological functioning well into adulthood (42). Likewise, Connolly and Mclsaac (21) verified that adolescent romantic encounters are initial steps on an experience toward a mature relationship to adulthood. As a result of these effects, romantic relationships can cause adolescents to feel or to be perceived as more mature, with enhanced responsibilities and self-image, and future expectations, including with regard to long-term relationships $(47,48)$. However, earlier studies found that adolescent intimate relationships with partners come with other risks, such as increased risk of STIs and unplanned pregnancy $(24,38)$.

\section{The power of SRH education}

There are several sources to provide SRH education, such as schools, the internet, friends, family, and health providers. Participants mainly learned about issues related to SRH such as puberty, contraception, sexual intercourse, becoming pregnant, and abstinence from school, healthcare professionals, or the internet. A study of adolescent students by Deshmukh and Chaniana (49), supported that the most common sources of information about SRH were teachers, mass media, and friends, while parents and siblings had the least important role. The role of school as a source of learning about SRH mainly relates to sex education/ health classes, which vary according to the age of learners, cultural expectations, and national policies. Various forms of sex education are commonly offered from the early years of elementary school in Western countries, while in most Asian countries it is provided at the high school level. Among university students, participants reported that they received reiterations of SRH education with which they were familiar from their high schools.

Deshmukh and Chaniana (49) reported that very few parents were brave enough to discuss reproductive health-related matters with their children at home, commonly due to a lack of awareness of reproductive health (60.75\%), and fear about encouraging premarital sex (51.40\%), aside from incidental shyness and 
stigma associated with sociocultural norms about discussing sexual issues with their own children. Notwithstanding, the current study found that parents openly communicated with their children concerning safe sex influenced decision-making in contraception use. Indeed, parental communication is a crucial effective tool for promoting healthy and safe sexual practices (49). Perhaps this related to socioeconomic status or education level that university students in Thailand are from privileged backgrounds, or their parents would be more educated and better equipped to discuss serious issue with their children.

This study also exposed that most participants obtained SRH education in high school and university, which they considered important for their sexual health and decision-making concerning contraceptive methods. Over the course of many years of gathering knowledge of SRH, they perceived benefits of SRH education to include better and more accurately information and a better understanding of body changes, alongside empowerment with skills such as negotiation (particularly for females), decision making, and communication, in order to protect themselves from unintended pregnancy and STIs. This is in line with a study of Vongxay, Albers (50), which concluded that comprehensive sexual education and enabling information, as well as service access for adolescents, is essential to ensure that adolescents can access, understand, appraise, and apply good SRH knowledge in decision-making for optimum personal health. Oonyu (51) supported this and determined that SRH education was necessary for female university undergraduate students in Uganda.

The accuracy of SRH education was reported to be particularly important among participants in shaping their sexual relationship choices and consistency of contraception use, consistent with previous studies which revealed that adolescent attitudes toward contraception and the accuracy of condom and reproductive knowledge directly influence contraceptive use throughout adulthood $(52,53)$. Conversely, Yared, Sahile (54) found that over half of university students in central Ethiopia knew about STIs but neglected to apply their knowledge to themselves and their sexual health. The majority of reproductive health problems in their study related to unwanted pregnancy and abortion caused by non-use of contraception. They thus suggested that SRH education should begin from high school, due to the fact that the majority of students in high school started sexual experience at an early age. Similarly, a study of Oonyu (51) in Uganda revealed that the majority of university students $(66.3 \%)$ requested SRH education to assist them to overcome barriers, such as an inability to get reliable and accurate information to empower them in decision making, and to overcome inadequate education from parents and the existing university provision.

The lack of accurate information coupled with low access to contraceptives may increase the risk of STIs, unintended pregnancy, and other health consequences $(53,55)$. Comprehensive SRH education and counselling could improve effective contraceptive behaviour throughout the life course (52). Therefore, SRH education is necessary for university students to solve decisions about relationships, sexuality, and sexual behaviours (51). However, the information transmitted about STIs and SRH via the media is commonly found to be boring by adolescents (54). It is suggested that such programmes should be youth-friendly, with separate packages targeted to STIs and SRH. 
Previous research indicates that perceived risks of pregnancy and STIs and motivation to avoid these outcomes are linked with adolescents' contraceptive method use $(51,52)$. In this study, older adolescents perceived that the risks of pregnancy and STIs were associated with contraceptive use. This reflects cognitive development, whereby late adolescents to link current behaviours with potential future consequences more strongly than younger adolescents and children. Morales, Vallejo-Medina (56) reinforced that adolescents in their study were more knowledgeable and displayed more favourable attitudes towards different aspects of HIV/AIDS. Adolescents who perceived greater risk and negative expectations about risky sex outcomes related to pregnancy and STI were more strongly influenced in their final decision to use of dual contraceptive methods $(51,52)$. Adolescents' attitudes about practical, social, and moral implications of using birth control are also associated with their final protective decisions (56).

\section{Parental attitudes regarding adolescent relationships}

In Thai tradition, having premarital sex is viewed with social disapprobation and disgrace. Premarital sex in adolescence was traditionally viewed with stigma in Thai society, but it has become increasingly normalised nowadays. This is because attitudes towards premarital sex reflect the changing nature of modern society, and such behaviours are now widely accepted in society. In previous studies, it seemed like adolescent sexuality and pregnancy were relatively normal phenomena in rural communities in Thailand $(24,57)$. Sriyasak, Almqvist $(57)$ reinforced that the changing of the dynamic social environment becomes the accepted in the traditional value in the North-eastern of Thailand. Notwithstanding, modernity is the factor that has stimatised adolescent pregnancy made it shameful in Thailand. As can be seen in many previous studies in Thailand found that pregnancy in adolescence is viewed as problematic and a serious social problem and pregnant adolescents are sensitive to being personally shamed by the words and gestures of people around them $(58,59)$

As this study found that parents play a major role in SRH of adolescents in Thailand, Leekuan (24) exposed that adolescents initial concealed their sexual relationship and pregnant from anybody, especially their parents because of being afraid of unaccepted sexual relationship and pregnancy from parents. In another research by Dalton (60) encouraged adolescents commonly hide sexual-related behaviour from their parents in the American context. This is in a line with Saim, Dufåker (61) revealed that Malaysian adolescents tried to hide their sexual relationship and their pregnancies from anybody.

Parents have always been the most important influence on adolescents' decisions about sex in many contexts, but parents commonly underestimate the impact they have on their decisions (62). This study also found that parents know about and have confidence in their daughters' decisions and activities. Surprisingly, this seems different from a study of Anyanwu, Akinsola (63) in the South Africa contexts found that African adolescents discomforted sharing their personal problems with their parents because they worried about their parents' forbidden dating. Generally, parenting tasks and responsibilities are an inherent part of raising children, and effectively promote their development. A study by Morales, VallejoMedina (56) in Colombia revealed adolescents believe that important people in their lives expect them to protect themselves during sexual intercourse, and most of them are willing to comply with these 
expectations. The relationships between parents and adolescents can also shape adolescent development (64).

Adolescents in this study recounted that their parents perceived their relationships and were concerned about sex-related issues, as well as birth control. Many previous studies presented similar findings, including parental perceptions of sexual relationships in adolescence (65). A study by Breuner, Mattson (66) identified that adolescents' perceptions of parental expectations about sex and contraception had important impacts on sexual activities, including the use of contraceptive methods.

Contrary to expectations, parents are more likely to talk with their adolescents about body changes and dating, rather than discussion about sex-related issues, birth control, and STIs (62). International research shows that the communication between adolescents and parents on issues such a sexual relationships, early pregnancy, HIV, and contraception is often very limited $(56,67,68)$. Because of a lack of knowledgeable and skills, as well as cultural norms and taboos concerning the discussion of sexual issues between parents and children, sexuality and sex education are commonly not discussed at home (68).

Previous research indicated that parental disapproval about adolescent sexual activity is an effective way to decrease the probability of adolescents engaging in sexually risky behaviours (69). Nonetheless, the findings in this study illustrated that the adolescents were more mature and could take responsibility, especially as they were studying at university with parental approval for their relationships with their partners. This suggests the great importance of adolescent responsibility and choices in such contexts, as Leekuan (24) studied cases of adolescent pregnancy and reported that although parents in many families consented to their adolescents cohabiting prior to marriage, and having sexual relationships, the adolescents did not give importance to protecting themselves from unintended pregnancy.

Although some of adolescents' parents approved having early premarital sexual relationship among adolescent, many of them concerned about these relationships. Often undisclosed of adolescents' sexual relationships in this study was revealed as being afraid of unaccepted relationship from parents and families. A study in Kenya of Maina, Ushie (70) revealed similar findings in which unpleasure occurrences about adolescent romantic relationship, it was covert and viewed as immoral as well as disturbed education goals. This is a line with a study of Killoren, Campione-Barr (71) found that American adolescents would choose to disclose partners and discuss thoughts and concerns related to sexual relationship to their mothers. However, a majority of them would not discuss thoughts and concerns related to dating with their mothers. Their research also reported that adolescents who had positive relationship qualities selected mothers as disclosure partners for sexuality.

\section{Conclusion}

One of the strengths of the present study is its updating of knowledge on SRH experience among older adolescents in Thailand. Reported data provide a better insight into the meaning of SRH experiences pertaining to the risks of unplanned pregnancies and STIs in this population. To the best of our 
knowledge, this is the first study to explore the experiences of SRH in older Thai adolescents. Data indicate that SRH education and parental perceptions about sex relationship in adolescents prevent unplanned pregnancy and the risks of STIs and promote a sense of intimacy and emotional closeness with the partners, which leads to greater well-being during the transition to adulthood.

Adolescence is a unique and critical phase in the lifespan, during which individuals attain sexual maturity. The current study uncovered parental perceptions about adolescents' sexual relationships are instrumental in preventing both unintended pregnancy and STIs. As a result of this, most participants made decisions to protect themselves with safe sex. Additionally, adolescent attitudes about practical, social, and moral implications of using birth control are also linked to their making decisions to use contraceptive methods. While the dimension of socio-economic status was not directly addressed among the studied variables, the fact that participants were university undergraduates implies that they are from families with relatively higher socio-economic status, which should be considered in interpreting the findings.

Sexual reproductive health contains all issues related to the reproductive system and its function. Sexual health indicates the ability of having a safe and satisfying sex life (72). SRH is also a human right and an achievement of the Millennium Development Goals (MDGs). SRH education is an influencing factor for adolescent pregnancy, child marriage, and other adverse SRH outcomes and negative social consequences, which occur more often in poor, less-educated, and rural communities such as Northern Thailand (73). This study exposed the connection of SRH education and making decisions about having sex safety. Therefore, SRH education should occur throughout the school journey, with information appropriate to students' age, religion, and cultural background. It should go beyond the current focus on biological aspects of sex and reproduction, and incorporate communities' attitudes, values, and skills.

Aside from schools and universities, SRH knowledge from parents was crucial in sexual education. Parents are the largest influence on their adolescents' decisions about sex, and they have profound impacts on their decisions. Participants in this study stated their parents told them to take care of themselves and prevent themselves from the risks of having sexual relationships. The prospect of talking about topics related to sexuality creates anxiety and apprehension, and this may lead to avoidance of such discussions (62). This underscores the critical role of educational access for the realization of SRH and rights for young people, especially girls (74).

Every adolescent has life-changing decisions to make about their SRH. The lack of sexuality education to make adolescent decisions responsibly can predispose adolescents to unintended adolescent pregnancy and STIs and leave females vulnerable to coercion. Lack of awareness of contraceptive use is also strongly associated with increased risk of early pregnancy. Disadvantaged adolescents are more inconsistent in their use of contraception, even when they do not want a pregnancy, and they tend to have more unintended pregnancies. A study in Thailand by Chirawatkul, Rungreangkulkij (75) found that adolescent women who achieve well in school and do not exhibit risky behaviours are more likely to become pregnant when they do not have sufficient knowledge and skills to protect themselves from such 
behaviours, and that adolescent men had low understanding of how contraceptive methods work. Adolescents' decisions and behaviours can have long-lasting implications, thus being ill-informed can have lifelong adverse consequences, both for themselves and (potentially) their children.

Romantic relationships which are characterised by intimacy and good communication can contribute to healthy adolescent growth, resilience, and happiness, and often provide a valuable foundation for longterm relationships into adulthood. However, while they are associated with healthy, normative development in most adolescents, they can be symptomatic of pathology in many others. Adolescent romantic relationships play an important role for the development of adolescence and encouraging directions for further well-being $(21,47)$.

Older adolescents in this study reported having had a special romantic relationship with their partners, which can support long-term relationships and promote taking responsibility. Their relationships with partners can also improve equality in emotion resources, sharing power in interaction and sharing decision-making responsibility. These current findings nuance the conclusion of earlier studies, in that romantic relationships have negative impacts on adolescent well-being in terms of dating in adolescence, depending on the surrounding context and norms (as well as individual factors, which are instrumental in every relationship). Engaging adolescent romantic and sexual relationship was closeness relationship as improving pleasure and life satisfaction as well as growing in adolescent development.

Based on the understandings of SRH experience in adolescents, this study addresses the gap between $\mathrm{SRH}$ experiences and adolescent development. Therefore, the $21 \mathrm{st}$ century is perceived not as a period of confused exploration, but rather as SRH experiences where adolescents gave meanings to facets of their lives relating to their relationships with sexual partners. Without this understanding, health education cannot move forward in clinical or educational interventions. Understanding SRH experience among late adolescents is also useful for interventions and helping healthcare professionals to understand and provide more effective interventions, helping guide adolescents during a critical life stage.

\section{Abbreviations}

AIDS

Auto-immune deficiently syndrome

ASRH

Adolescent sexual and reproductive health

HIV

Human Papilloma Virus

LMICs

Low-income and middle-income countries

MDGs

Millennium Development Goals

SDG 
Sustainable development goals

$\mathrm{SRH}$

Sexual and Reproductive Health

STI(s)

Sexually transmitted infection(s)

WHO

World Health Organization

\section{Declarations}

\section{Acknowledgements}

The authors are very grateful to all the participants in this study.

\section{Authors' contributions}

LP and SP designed the study. LP, SP and KW collected the data. LP, SP and KW interviewed and participated in intellectual data analysis. LP drafted and KR and SP revised the manuscript. All authors read and approved the final manuscript.

\section{Funding}

School of Nursing, University of Phayao funded the study, with grant reference no. BNS 256202003

\section{Data availability}

The dataset used and analysed during this study are available from the corresponding author on reasonable request.

\section{Consent for publication}

Informed consent was obtained from all participants involved in the study. No personal data are included. All quotations from the dataset have been anonymized.

\section{Ethics approval and consent to participate}

Ethical approval to conduct this study was granted by the University of Phayao Human Ethics Committee (Thailand) prior to commencing data collection. (No. 3/026/62)

\section{Conflict Interests}

The authors declared no potential conflicts of interest with respect to the research, authorship, and/or publication of this article. 


\section{References}

1. UNICEF. The adolescent brain: vulnerability and opportunity 2015 [Available from: https://www.unicef-irc.org/article/1149-the-adolescent-brain-vulnerability-and-opportunity.html.

2. UNHCR. Child and Adolescent Development Module: Action for the Rights of Children (ARC) 2018 [Available from: https://www.unhcr.org/3bb81bad4.pdf.

3. UNFPA. UNFPA strategy on Adolescents and Youth. 2013 [Available from: https://www.unfpa.org/resources/unfpa-strategy-adolescents-and-youth.

4. National Academies of Sciences E, and Medicine. The Promise of Adolescence: Realizing Opportunity for All Youth. Washington, DC: The National Academies Press; 2019.

5. WHO. WHO recommendations on adolescent sexual and reproductive health and rights. 2018 [Available from: https://apps.who.int/ iris/bitstream/handle/10665/275374/9789241514606eng.pdf?ua=1.

6. WHO. Sexual health and its linkages to reproductive health: an operational approach 2017 [Available from: https://apps.who.int/iris/bitstream/handle/10665/ 258738/9789241512886-eng.pdf..

7. WHO. Developing sexual health programmes: a framework for action 2010 [cited 202116 May]. Available from: http://www.who.int/reproductivehealth/ publications/sexual_health/rhr_ hrp_10_22/en/.

8. UN. Reproductive rights are human rights: a handbook for national human rights institutions 2014 [cited 2021. Available from: http://www. ohchr.org/Documents/Publications/NHRIHandbook.pdf.

9. WHO. Sexual health, human rights and the law 2015 [cited 2021. Available from: http://www.who.int/reproductivehealth/publications/ sexual_health/sexual-health-human-rightslaw/en/.

10. WHO. Regional consultation on the development of the European action. plan for sexual and reproductive health and rights (SRHR) 2017-2021: Copenhagen, Denmark, 14-15 December 2015 2016 [cited 2021. Available from: http://www.euro.who.int/_data/assets/pdf_ file/0008/300122/Regional-consultation-development-EAP-SRHR20172021-report.pdf.

11. WHO. Sexual and Reproductive Health and Research (SRH). 2021 [Available from: https://www.who.int/teams/sexual-and-reproductive-health-and-research-(srh)/about-us.

12. Bureau of Reproductive Health MoPH. Situations reproductive health in adolescents and youth in 20182018 [Available from: http://rh.anamai. moph.go.th/ download/all_file/ index/situationRH_ 2561_Website.pdf.

13. Santhya K, Jejeebhoy SJ. Sexual and reproductive health and rights of adolescent girls: Evidence from low-and middle-income countries. Glob Public Health. 2015;10(2):189-221.

14. UNFPA. Programme of Action - Adopted at the International Conference on Population and Development (ICPD). Cairo 1994 [cited 202123 August]. Available from: https://www.unfpa.org/sites/default/files/event-pdf/PoA_en.pdf. 
15. UNFPA. International Conference on Population and Development 2021 [cited 202112 January]. Available from: https://www.unfpa.org/icpd.

16. UNICEF Thailand. A SITUATION ANALYSIS OF ADOLESCENTS IN Thailand 2015-2016. Thailand; 2016.

17. National AIDS Committee. Thailand National Strategy to End AIDS 2017-2030 Bangkok, Thailand; 2017 April 2017.

18. Yau S, Adamu Y, Wongsawat P, Songthap A. Prevalence and correlates of contraceptive use among vocational schools adolescents in Northern Thailand. Journal of Health Research. 2021.

19. Holness NA. The Effects of Resilience and Social Influences on Preventing Repeat Adolescent Pregnancies in Parenting Adolescent Mothers. Florida International University.; 2014.

20. Hazen E, Schlozman S, Beresin E. Adolescent psychological. Pediatr Rev. 2008;29(5):161.

21. Connolly J, Mclsaac C. Adolescents' explanations for romantic dissolutions: A developmental perspective. J Adolesc. 2009;32(5):1209-23.

22. Tammilehto J, Punamäki R-L, Flykt M, Vänskä M, Heikkilä LM, Lipsanen J, et al. Developmental Stage-Specific Effects of Parenting on Adolescents' Emotion Regulation: A Longitudinal Study From Infancy to Late Adolescence. Frontiers in psychology. 2021;12.

23. Dombola GM, Manda WC, Chipeta E. Factors influencing contraceptive decision making and use among young adolescents in urban Lilongwe, Malawi: a qualitative study. Reproductive Health. 2021;18(1):1-11.

24. Leekuan P. Understanding adolescent pregnancy from the perspectives of pregnant adolescents in a Northern Thailand province: A phenomenological study. UK: University of Nottingham; 2020.

25. Sridawruang C, Crozier K, Pfeil M. Attitudes of adolescents and parents towards premarital sex in rural Thailand: A qualitative exploration. Sex Reproductive Healthc. 2010;1(4):181-7.

26. Beres MA, Pearman-Beres LJ, Johns P. Youth healthy and safe relationships: A literature review. 2020.

27. Chanthasukh S. Decision making and contraception: Perceptions and experiences of Thai-Isan adolescents. Auckland, University of Technology; 2019.

28. Heidegger M. Being and Time (MacQuaeeie J. \& Robinson E. trans). New York: Harper \& Row; 1962.

29. Heidegger M. Being and Time. New York: State University of New York Press; 2010.

30. UNICEF. Adolescent health and development. 2020 [cited 202028 April ]. Available from: https://www.unicef.org/thailand/topics/adolescent-health-and-development?items_ per _ page $=10 \&$ page $=2$.

31. National Academies of Sciences E. Medicine. The promise of adolescence: Realizing opportunity for all youth. National Academies Press; 2019.

32. Herwandha KG, Prastuti E. Attachment and Age As Predictors of the Emotional Maturity of University Students. KnE Social Sciences. 2020:1-20-1\&\#8211. 
33. Arnett JJ. Emerging adulthood: A theory of development from the late teens through the twenties. Am Psychol. 2000;55(5):469.

34. Packer MJ. Tracing the hermeneutic circle: Articulating an ontical study of moral conflicts. Entering the circle: Hermeneutic investigation in psychology. 1989:95-117.

35. Packer MJ. Hermeneutic inquiry in the study of human conduct. 1992.

36. van Manen M. From meaning to method. Qualitative health research. 7, editor1997.

37. Smith JA, Flowers P, Larkin M. Interpretative phenomenological analysis: Theory, method and research. London: SAGE publications, Ltd.; 2009.

38. Salvador JT, Sauce BRJ, Alvarez M, Rosario A. The phenomenon of teenage pregnancy in the Philippines. Eur Sci J. 2016;12:32.

39. Nkurunziza A, Van Endert N, Bagirisano J, Hitayezu JB, Dewaele S, Tengera O, et al. Breaking barriers in the prevention of adolescent pregnancies for in-school children in Kirehe district (Rwanda): a mixed-method study for the development of a peer education program on sexual and reproductive health. Reproductive Health. 2020;17(1):1-8.

40. Gómez-López M, Viejo C, Ortega-Ruiz R. Psychological well-being during adolescence: Stability and association with romantic relationships. Front Psychol. 2019;10:1772.

41. Price M, Hides L, Cockshaw W, Staneva AA, Stoyanov SR. Young love: Romantic concerns and associated mental health issues among adolescent help-seekers. Behav Sci. 2016;6(2):9.

42. Kansky J, Allen JP. Long-term risks and possible benefits associated with late adolescent romantic relationship quality. J Youth Adolesc. 2018;47(7):1531-44.

43. Furman W, Low S, Ho MJ. Romantic experience and psychosocial adjustment in middle adolescence. J Clin Child Adolesc Psychol. 2009;38(1):75-90.

44. Roisman GI, Masten AS, Coatsworth JD, Tellegen A. Salient and emerging developmental tasks in the transition to adulthood. Child Dev. 2004;75(1):123-33.

45. Putri DAWM, Yuniarti KW, Minza WM, Riyono B. How Balinese Adolescents Perceived Romantic Relationship. J Family Sci. 2020;5(2):71-91.

46. Wheeler LA, Killoren SE, Whiteman SD, Updegraff KA, McHale SM, Umaña-Taylor AJ. Romantic relationship experiences from late adolescence to young adulthood: The role of older siblings in Mexican-origin families. J Youth Adolesc. 2016;45(5):900-15.

47. Shulman S, Connolly J. The challenge of romantic relationships in emerging adulthood: Reconceptualization of the field. Emerg Adulthood. 2013;1(1):27-39.

48. Hensel DJ, Nance J, Fortenberry JD. The association between sexual health and physical, mental, and social health in adolescent women. J Adolesc Health. 2016;59(4):416-21.

49. Deshmukh DD, Chaniana SS. Knowledge About Sexual and Reproductive Health in Adolescent School-Going Children of 8th, 9th, and 10th Standards. J Psychosexual Health. 2020;2(1):56-62.

50. Vongxay V, Albers F, Thongmixay S, Thongsombath M, Broerse JE, Sychareun V, et al. Sexual and reproductive health literacy of school adolescents in Lao PDR. PLoS ONE. 2019;14(1):e0209675. 
51. Oonyu J. IS SEXUAL REPRODUCTIVE HEALTH EDUCATION FOR UNIVERSITY STUDENTS NECESSARY?, THE CASE OF UNDERGRADUATE FEMALE STUDENTS'AT MAKERERE UNIVERSITY. UGANDA Ethiop J Reproductive Health. 2019;11(2):9-.

52. Guzzo KB, Lang VW, Hayford SR. Teen Girls' Reproductive Attitudes and the Timing and Sequencing of Sexual Behaviors. J Adolesc Health. 2019;65(4):507-13.

53. Guzzo KB, Hayford SR. Adolescent Reproductive Attitudes and Knowledge Effects on Early Adult Unintended and Nonmarital Fertility Across Gender. Advances in Life Course Research. 2021:100430.

54. Yared A, Sahile Z, Mekuria M. Sexual and reproductive health experience, knowledge and problems among university students in Ambo, central Ethiopia. Reproductive health. 2017;14(1):1-7.

55. Guzzo KB, Hayford SR, Lang VW, Wu H-S, Barber J, Kusunoki Y. Dimensions of reproductive attitudes and knowledge related to unintended childbearing among US adolescents and young adults. Demography. 2019;56(1):201-28.

56. Morales A, Vallejo-Medina P, Abello-Luque D, Saavedra-Roa A, García-Roncallo P, Gomez-Lugo M, et al. Sexual risk among Colombian adolescents: knowledge, attitudes, normative beliefs, perceived control, intention, and sexual behavior. BMC Public Health. 2018;18(1):1-13.

57. Sriyasak A, Almqvist A-L, Sridawruang C, Neamsakul W, Häggström-Nordin E. Struggling with motherhood and coping with fatherhood-A grounded theory study among Thai teenagers. Midwifery. 2016;42:1-9.

58. Neamsakul W. Unintended Thai adolescent pregnancy: A grounded theory study. US: University of California, San Francisco.;; 2008.

59. Ounjit W. Pre-marital sex and pregnancy: The high price of forgiveness. Int J Social Sci Humanity. 2011;1(2):111.

60. Dalton ED. Communication, control, and time: the lived experience of uncertainty in adolescent pregnancy. 2014.

61. Saim NJ, Dufåker M, Ghazinour M. Teenagers' experiences of pregnancy and the parents' and partners' reactions: a Malaysian perspective. J Family Violence. 2014;29(4):465-72.

62. Ashcraft AM, Murray PJ. Talking to parents about adolescent sexuality. Pediatr Clin North Am. 2017;64(2):305.

63. Anyanwu FC, Akinsola HA, Tugli AK, Obisie-Nmehielle N. A qualitative assessment of the influence of family dynamics on adolescents' sexual risk behaviour in a migration-affected community. Int $J$ qualitative Stud health well-being. 2020;15(1):1717322.

64. Baumrind D. Parental disciplinary patterns and social competence in children. Youth \& Society. 1978;9(3):239-67.

65. Oluyemi JA, Yinusa MA, Abdullateef R, Kehinde K, Adejoke J. Parental influence on adolescent sexual behaviour among secondary school students in Ogbomoso, Nigeria. Afr J Social Work. 2017;7(1):37-43. 
66. Breuner CC, Mattson G, Child CoPAo, Health F. Sexuality education for children and adolescents. Pediatrics. 2016;138(2).

67. Isaksen KJ, Musonda P, Sandøy IF. Parent-child communication about sexual issues in Zambia: a cross sectional study of adolescent girls and their parents. BMC Public Health. 2020;20(1):1-12.

68. Ram S, Andajani S, Mohammadnezhad M. Parent's perception regarding the delivery of sexual and reproductive health (SRH) education in secondary schools in Fiji: A qualitative study. Journal of environmental and public health. 2020;2020.

69. Kincaid C, Jones DJ, Sterrett E, McKee L. A review of parenting and adolescent sexual behavior: The moderating role of gender. Clin Psychol Rev. 2012;32(3):177-88.

70. Maina BW, Ushie BA, Kabiru CW. Parent-child sexual and reproductive health communication among very young adolescents in Korogocho informal settlement in Nairobi, Kenya. Reproductive Health. 2020;17:1-14.

71. Killoren SE, Campione-Barr NM, Jones SK, Giron SE. Adolescent Girls' Disclosure About Dating and Sexuality. J Fam Issues. 2019;40(7):887-910.

72. WHO. The importance of sexual and reproductive health and rights to prevent HIV in adolescent girls and young women in eastern and southern Africa. 2017 [Available from: https://apps.who.int/iris/bitstream/handle/ 10665/ 255334/WHO-RHR-17.05-eng.pdf?sequence=1.

73. UNFPA. How our future depends on a girl at this decisive age. State of world population 2016; Chap 1-3 2016 [Available from: https://www.unfpa.org/swop-2016.

74. Starrs AM, Ezeh AC, Barker G, Basu A, Bertrand JT, Blum R, et al. Accelerate progress-sexual and reproductive health and rights for all: report of the Guttmacher-Lancet Commission. The Lancet. 2018;391(10140):2642-92.

75. Chirawatkul S, Rungreangkulkij S, Jong-Udomkarn D, Sawangchareon K, Anusornteerakul S, Wattananukulkiat $S$, et al. Prevention of and dealing with teenage pregnancy. Bangkok: Bureau of Gender Equity Promotion, Office of Women's Affairs and Family Development; 2011.

\section{Supplementary Files}

This is a list of supplementary files associated with this preprint. Click to download.

- COREQChecklist11.pdf 